\title{
Potential Treatment for Multiple Myeloma: Immune Checkpoint Inhibitors
}

\author{
Jiaxin Wang, Jiaxiong Tan, Yuhong Lu* \\ Department of Hematology, First Affiliated Hospital, Jinan University, Guangzhou, China \\ Email address: \\ merryveraforever@163.com (Jiaxin Wang),gdydtjx@163.com (Jiaxiong Tan),yhl2006jn@163.com (Yuhong Lu) \\ ${ }^{*}$ Corresponding author
}

\section{To cite this article:}

Jiaxin Wang, Jiaxiong Tan, Yuhong Lu. Potential Treatment for Multiple Myeloma: Immune Checkpoint Inhibitors. International Journal of Immunology. Vol. 9, No. 3, 2021, pp. 59-67. doi: 10.11648/j.iji.20210903.14

Received: September 3, 2021; Accepted: September 17, 2021; Published: September 26, 2021

\begin{abstract}
Multiple myeloma (MM) is a malignant disease in which monoclonal plasma cells proliferate abnormally. The poor prognosis of MM is always closely related to the immunosuppression of $\mathrm{T}$ cells. Restore the immune function of suppressed $\mathrm{T}$ cells may reverse the immunodeficiency in the $\mathrm{MM}$ microenvironment and improve prognosis. In recent years, immunosuppressive receptors such as programmed cell death receptor-1 (PD-1), T cells immunoglobulin and ITIM domain (TIGIT), Lymphocyte-Activation Gene-3 (LAG-3), T cell immunoglobulin and mucin domain-containing molecule 3 (Tim-3), Leukocyte immunoglobulin-like receptor B1 (LILRB1) and Cytotoxic T- lymphocyte antigen 4 (CTLA-4) have been discovered playing a key role in the tumor immunodeficiency microenvironment. For patients with solid tumors and some leukemia, immunotherapy targeting such receptors can significantly improve the $T$ cells immunodeficiency. However, similar positive results were not found in MM patients, which is related to the complex immunosuppressive mechanism. At present, the understanding of immunosuppressive receptors in MM is insufficient. In this review, this paper summarized part of the studies on PD-1, TCLA-4, Tim-3, TIGIT and other popular immunosuppressive receptors in MM, in order to attract more attention, and in-depth research on the immunotherapy also to promote the immunotherapy of MM from basic research to clinical transformation as soon as possible.
\end{abstract}

Keywords: Multiple Myeloma, Immune Therapy, Immunosuppressive Receptors, T Cell Exhaustion, Immune Dysfunction

\section{Introduction}

Multiple myeloma (MM) is a malignant disease caused by the abnormal proliferation of monoclonal plasma cells. In recent years, the promotion and application of proteasome inhibitors and immunomodulators have prolonged the survival of MM patients, but MM is still an incurable disease. Studies have shown that the poor prognosis of MM is closely related to the immune suppression of $\mathrm{T}$ cells. Reversing $\mathrm{T}$ cell immunodeficiency may lead to a better prognosis. [1] It has been reported that there are immune dysfunction mechanisms such as abnormal $\mathrm{T}$ cell classification ratio, low cytotoxicity and functional exhaustion in MM patients [2]. However, conventional treatments can lead to premature aging phenotypes and metabolic dysfunction in $\mathrm{T}$ cells, which makes it even more difficult for MM patients with existing immunodeficiency to restore the immune function of
$\mathrm{T}$ cells [3]. Recent in-depth studies on tumor microenvironment have shown that immunosuppressive receptors such as programmed cell death receptor-1 (PD-1) [4], T cell immunoglobulin and ITIM domain (TIGIT) [5], Lymphocyte-Activation Gene 3 (LAG-3) [6], T cell immunoglobulin and mucin domain-containing molecule 3 (Tim-3) [7], Leukocyte immunoglobulin-like receptor B1 (LILRB1) [8] and cytotoxic T- lymphocyte antigen 4 (CTLA-4) [9] play a key role in the formation of an immunosuppressive microenvironment. Clinical studies have shown that targeted immunosuppressive receptor immunotherapy for patients with solid tumors and some leukemias can significantly improve the $T$ cell immunodeficiency of patients and achieve exciting clinical effects [10-12].

The above results provide hope for MM patients who are refractory to treatment and prone to relapse. However, clinical studies on MM patients have not shown positive 
results. The reason may be that there are multiple immunosuppressive receptors involved in mediating tumor immunity in MM patients. Targeting a single immunosuppressive receptor cannot well reverse $\mathrm{T}$ cell immune dysfunction. Therefore, this article will review the research of some common immunosuppressive receptors in $\mathrm{MM}$, aiming to provide more basic information and strategies for the combined anti-immunosuppressive receptor therapy of MM

\section{Immunosuppressive Receptor}

\section{1. $P D-1$}

PD-1 is a member of the CD28 familyinhibit $\mathrm{T}$ cell activation by inhibiting CD28 costimulation [13]. Under normal circumstances, the combination of PD-1 and PDL1/2 can transmit inhibitory signals, maintain the immune tolerance of peripheral lymphocytes to self-antigens, and avoid the occurrence of immune storms [14]. However, in tumor environments such as lymphoma, non-small cell lung cancer and acute myeloid leukemia, the overexpression of PD-1 and PD-L1/2 is an important factor in tumor progression and poor prognosis $[4,15,16]$. Studies have found that MM patients have an imbalance and dysfunction in the ratio of CD4 to CD8 $\mathrm{T}$ cell subsets, which mediate reduced cytotoxicity, restricted $\mathrm{T}$ cells cloning, and suppressed $\mathrm{T}$ cells activation $[17,18]$. Therefore, studying the causes of immunodeficiency in MM patients is a prerequisite for immunotherapy.

Our previous studies found that the ratio of PD- $1+\mathrm{CD} 3+\mathrm{T}$ cells in the bone marrow of MM patients was higher than peripheral blood, which accompanied by positive expression of depletion phenotypes CD244 and CD57 [2]. Suggesting that $\mathrm{T}$ cells were in the state of depletion under the tumor microenvironment, and PD-1 is likely to be involved in the exhaustion of CD3 $+\mathrm{T}$ cells in the bone marrow of MM. In addition, it has also been reported that the PD-1/PD-L1 pathway involves not only $\mathrm{T}$ cells, but also tumor-related DC cells and NK cells [19]. Researchers found high expression of PD-L1 in the DC cells subsets of the bone marrow of MM patients. The above suggests that the PD-1/PD-L1 pathway may weaken the ability of DC cells to trigger T cell responses, thereby mediating tumor cell immune escape [20]. Anything else, researchers such as Benson found that the NK cells of healthy people do not express PD-1, while the NK cells of MM patients highly express PD-1 [21]. In vitro experiments, the anti-PD-1 antibody was used to inhibit PD-1/PD-L1 signaling pathway, which can significantly improve the function of NK cells to kill MM cells [21]. The above confirm that PD-1/PD-L1 is overexpressed in a variety of immune cells in MM patients, and targeting this pathway may reverse the immunodeficiency state of MM patients.

Subsequently, PD-L1 inhibitors were applied to MM mouse models for the first time. This animal experiment showed that it can improve immunity and increase the survival rate of MM mice from $0 \%$ to $40 \%$ [22]. In vitro experiments and animal experiments showed the feasibility of targeting PD-1/PD-L1 to reverse MM immunodeficiency. However, in a Ib clinical trial, nivolumab was used as a single drug to treat 27 patients with relapsed or refractory multiple myeloma, of which only 1 patient obtained CR [23]. In another phase II clinical trial, pembrolizumab, pomalidomide and dexamethasone combined treatment for relapsed and refractory MM, only $8 \%$ of patients were close to CR, 19\% VGPR and 33\% PR [24]. Obviously, the treatment of PD-1 monoclonal antibody has not achieved satisfactory clinical efficacy. Based on the study of the tumor immune microenvironment of MM patients, the reason for the failure of PD-1 monoclonal antibody immunotherapy may be related to the complex $\mathrm{T}$ cell immunosuppressive mechanism of MM. In addition, our previous study also found a higher proportion of $\mathrm{PD}-1+\mathrm{Tim}-3+\mathrm{CD} 8+\mathrm{T}$ cells accompanying an increased exhaustion phenomenon of CD57 in the bone marrow of $\mathrm{MM}$ patients, pointed out that besides the PD1/PD-L1 pathway there are still other pathway involved in the exhaustion of $\mathrm{T}$ cell function in MM [2].

Besides studying the role of targeting PD-1/PD-L1, some researchers have found in the treatment of non-small cell lung cancer, in that the clinical response of anti-PD-1 immunotherapy does not depend on the detection of PD1/PD-L1 level, but is positively correlated with the burden of non-synonymous mutations in the patient's somatic cells [25]. The above studies highlight the role of somatic mutation in anti-PD-1 immunotherapy. Moreover, there are data showing that the expression of PD-L1 in different locations in the tumor microenvironment such as infiltrating immune cells, infiltration margins, tumor core, etc. may affect the function of PD-L1 as a biomarker [26, 27]. The above all indicate that the burden of somatic mutation and the distribution of immunosuppressive molecules in the tumor microenvironment are equally important in anti-PD-1 immunotherapy, and these aspects in MM still need to be further studied.

\subsection{CTLA-4}

Cytotoxic T- lymphocyte antigen 4 (CTLA-4) is a homolog of CD28, mainly expressed in CD4+T and CD8+T cells, and constitutive expressed on Treg cells. Antigen achieves immune escape by CTLA-4 binding to the ligand B7 molecule to transmit signals that inhibit $\mathrm{T}$ cell proliferation and cytotoxicity $[9,28]$.

In the study of solid tumors [29, 30], it was found that overexpression of CTLA-4 represents a poor prognosis and disease progression. In hematological malignancies, CTLA-4 may also be a prognostic marker for patients with AML, MDS, and lymphoma $[31,32]$. It is worth noting that the overexpression of FOXP3 and CTLA4 in bone marrow samples found in the MM patients suggests that local accumulation of Tregs is immunosuppressed [28]. Blocking the expression of CTLA-4 may reverse the immune deficiency in the tumor microenvironment to some extent.

In 2010, a phase III randomized controlled clinical trial showed that the use of the anti-CTLA4 antibody Ipilimumab to treat patients with metastatic melanoma can prolong survival [33]. Since then, Ipilimumab has obtained varying 
degrees of benefit in the treatment of many solid tumors, such as non-small cell lung cancer and kidney cancer [34, 35]. For the treatment of MM, a phase I/Ib trial is currently underway to study the side effects and optimal dose of Ipilimumab or Nivolumab in patients with hematological malignancies recurring after stem cell transplantation (NCT01822509).

Although PD-1 is also one of the mechanisms that cause $\mathrm{T}$ cells exhaustion, CTLA-4 is different from PD1, in that CTLA-4 acts in the initial stage of naive T cells activation, usually in the lymph nodes [36]. The PD-1 pathway inhibits T cells in the late stage of immune response, mainly in peripheral tissues [37]. The above suggests that PD-1 and CTLA-4 inhibit T cells at different times, and it may be better to inhibit the expression of both receptors at the same time. In non-small cell lung cancer (NSCLC) with high expression of wild-type EGFR and CTLA4, anti-CTLA-4 antibody can induce the expression of PD-L1 in NSCLC cells [38]. The combined effect of anti-CTLA4 and anti-PD-1 antibody is better than anti-PD-1 antibody alone [38]. The reason for this result may be that two different antibodies in different molecular pathways jointly relieve the immune suppression of $\mathrm{T}$ cells, so the combined application can show a better effect of activating cellular immunity. This conclusion is also confirmed in the treatment of solid tumors [39, 40], that the combined blockade of CTLA-4 and PD1 does extend the survival of tumor patients, which brings new hope for the treatment of MM.

Studies have also found that EGFR is a key protein that causes exosomes to promote resistance to bortezomib in myeloma cells [41]. Combination therapy of anti-EGFR aptamer and Ipilimumab has achieved encouraging results in the treatment of non-small cell lung cancer [42]. It can be inferred that if the above combined treatment is adopted, it can not only improve the efficacy of anti-PD-1, but also improve the resistance of MM cells to proteasome inhibitor. However, it should be noted that studies have found that blocking CTLA-4 can promote the proliferation of lung cancer cells, which is a bad signal for anti-tumor therapy. More clinical evidence of anti-CTLA-4 in MM is needed to prove the safety.

\subsection{TIGIT}

$\mathrm{T}$ cell immunoglobulin and ITIM domain (TIGIT) are members of the CD28 family, a new type of co-inhibitory receptor, which is mainly expressed on the surface of NK cells. The combination of TIGIT with its CD155 (PVR) and CD112 (PVRL2, nectin-2) ligands directly inhibits the activation of NK cells $[43,44]$. As the costimulatory receptor of TIGIT, CD226 can also bind to CD155, but has the opposite effect to TIGIT, and TIGIT binds to CD155 with higher affinity to inhibit CD226 signal transduction, thereby reducing the anti-tumor immune effect of NK cells [45]. Studies have found that the overexpression of TIGIT and PD-1 on the surface of NK cells and T cells and the down-regulation of CD226 expression can also lead to the reduction of the secretion of anti-tumor cytokines such as CD107a, INF- $\gamma$ and TNF- $\alpha[5]$.

In solid tumors $[46,47]$, the TIGIT ligands CD155 and
CD112 are overexpressed, which seems to indicate a certain association between tumors and overexpressed TIGIT. The abnormal expression of TIGIT also occurs in hematological malignancies. First, T cells and NK cells of MDS patients show that the expression level of CD226 is reduced, and the expression level of TIGIT is increased [5]. Secondly, Guillerey et al. found in the body of MM tumor-bearing mice that $30-40 \%$ of Treg cells in the bone marrow express TIGIT, and reported in mouse and human studies that the progress of MM is related to the high level of TIGIT expression on CD8+ $\mathrm{T}$ cells. Relatedly, the bone marrow CD8+ T cells of newly diagnosed or relapsed MM patients express higher levels of TIGIT than the healthy, NK cells and CD4+ T cells express intermediate levels of TIGIT [44]. The above also indicate that in MM, TIGIT is mainly expressed on CD8+ T cells, which reduces the body's anti-tumor activity by inhibiting the activation of lymphocytes effect. Subsequently, Guillerey confirmed in a mouse model that TIGIT can limit the immune response to myeloma, and monoclonal antibodies can be used to block TIGIT receptors to increase the activation of $\mathrm{CD} 8+\mathrm{T}$ cells in MM mice and inhibit the progression of MM [44].

It is widely known that autologous stem cell transplantation (ASCT) is a common clinical method for the treatment of refractory relapsed and high-risk MM. Although some patients can obtain short-term clinical remission after transplantation, tumor recurrence and disease progression are still inevitable. Animal experiments found that in mice with $\mathrm{MM}$ recurrence after ASCT, in the bone marrow the expression ratio of $\mathrm{CD} 226$ in $\mathrm{CD} 8+\mathrm{T}$ cells decreased, while the expression ratio of other immunosuppressive receptors increased, such as TIGIT, PD-1, LAG- 3, Tim-3, etc. and the expression of inhibitory receptors on $\mathrm{CD} 8+\mathrm{T}$ cells is related to myeloma load [48]. While researchers have found that the inhibition of TIGIT early after transplantation can prevent $\mathrm{T}$ cell failure, thereby maintaining long-term immune control of myeloma, and show fewer adverse reactions than PD-1 checkpoint inhibitors [49]. The above studies suggest that targeting immunosuppressive receptors TIGIT may be possible to further improve the prognosis of MM patients who undergoing ASCT.

Moreover, targeting PD-1 combined with TIGIT has obtained pleasing results in experiments. Dual TIGIT and PD-1/PD-L1 blockade can work synergistically to enhance the proliferation of anti-tumor CD8+ T cells function [50-52]. In mouse tumor models and in vitro experiments, after dual PD-1/TIGIT blockade [50], it was found that the inhibitory effect of the immune environment on CD226 disappeared, which shows that blocking TIGIT can activate the promotion of CD226 on immune cells. In clinical trials, the currently commonly used anti-TIGIT antibody is Tiragolumab. The results of a phase II study (NCT03563716) of Tiragolumab and PD-L1 inhibitor (Tecentriq) in the treatment of PD-L1+ advanced NSCLC showed that despite similar toxicity, double PD-L1 /TIGIT blockade seems to provide better clinical efficacy than PD-L1 blockade alone as the first-line treatment of PD-L1+ non-small cell lung cancer. Regarding the treatment of MM, the current clinical trial is a phase I/II 
randomized trial aimed at patients with relapsed refractory multiple myeloma, aiming to evaluate two immune checkpoint inhibitors' (anti-LAG-3 and anti-TIGIT) immunological effect and safety (NCT04150965).

The role of anti-TIGIT antibodies in myeloma has not been discussed much in the clinical setting. Due to the lack of more preclinical animal validation, no direct evidence was provided to support the clinical role of anti-TIGIT antibodies in MM patients. Therefore, future research should focus on testing the effectiveness and tolerability of anti-TIGIT antibodies in living models before applying the results to the clinic.

\section{4. $L A G-3$}

Lymphocyte activating gene-3 (LAG-3) is a member of the immunoglobulin (Ig) superfamily [6]. It is expressed in activated $\mathrm{T}$ cells, NK cells, $\mathrm{B}$ cells and plasmacytoid dendritic cells (pDCs), mainly through binding to MHC II molecules to down-regulate the activity of $\mathrm{T}$ cells and also enhance the inhibitory activity of Treg cells [53]. In tumor immunity, LAG-3 has been identified as one of the main inhibitory receptors involved in T lymphocyte exhaustion in tumor diseases, and its inhibitory function is closely related to its expression level on the surface of T lymphocytes [54]. In malignant diseases or chronic inflammation, continuous $\mathrm{T}$ cell activation will cause LAG-3 and other inhibitory receptors to be continuously co-expressed on $\mathrm{T}$ cells [55] which can explain why a single anti-PD-1 treatment cannot achieve the desired therapeutic effect and causes treatment resistance [56]. In addition, the inhibitory function of LAG-3 also includes the inhibition of $\mathrm{T}$ cell proliferation and the production of anti-tumor cytokines, including INF- $\gamma$ and TNF $\alpha$ [57].

The expression of LAG-3, like other immunosuppressive receptors, is increased in a variety of solid tumors [58, 59]. The overexpression of LAG-3 has also been found in studies of hematological tumors. For example, the expression of CTLA-4 and LAG-3 genes in AML patients are significantly up-regulated [60], and the expression of LAG-3 on CD8+ T and regulatory $\mathrm{T}$ cells in MDS patients is higher than healthy people [61]. During the transformation from monoclonal gamma globulinemia to $\mathrm{MM}$, the expression of PD-L1 on plasma cells and the expression of LAG-3 in the microenvironment increased [62]. Besides, it was found that an increased expression of LAG-3- in peripheral blood $\mathrm{T}$ lymphocyte (PBTL) in 90 days after ASCT was an early indicator of relapse or death in MM [63]. The above studies suggest that the abnormal expression of LAG-3 promotes the proliferation of minimal residual tumor cells for patients whose immune function has not been reconstructed after ASCT, and may be an important predictor of poor prognosis in MM patients. Target blocking the inhibitory effect of LAG-3 in various immune cells may restore the anti-tumor function.

Early studies have found that the combination of LAG-3 blockers and specific anti-tumor vaccines can significantly increase the number of activated $\mathrm{CD} 8+\mathrm{T}$ cells in the tumor microenvironment and destroy the tumor substance [64]. In a mouse model, anti-LAG-3 antibody treatment delayed tumor growth [65]. The combination of anti-PD-1 antibody and anti-LAG-3 antibody has a better inhibitory effect on tumor growth than monotherapy, which is consistent with previous conclusions [66]. In animal experiments, it was first discovered that the combination of PD-L1 inhibitors and LAG-3 inhibitors can indeed improve the survival rate of myeloma mouse models $[62,67]$. This seems to have found another potential pathway for the treatment of MM.

In current clinical research on anti-LAG-3 drugs, a phase I/II clinical trial reported that the vaccination of LAG-3Ig (IMP321) and peptides can induce specific CD4+ and CD8+ $\mathrm{T}$ cell responses in patients with metastatic melanoma [68]. Other clinical trials of anti-LAG-3 antibodies are ongoing, and no specific results have been announced yet. Such as the safety study of anti-LAG-3 in relapsed or refractory hematological malignancies, the combination of anti-LAG-3 antibody and anti-TIGIT antibody (NCT04150965), anti-LAG-3 combined with or without anti-PD-1 in research in the treatment of solid tumors (NCT01968109) and so on. Regarding the specific use of anti-LAG-3 drugs, more clinical trials are still needed to prove its safety and effectiveness.

\subsection{Tim-3}

$\mathrm{T}$ cell immunoglobulin and protein 3 containing mucin domain (Tim-3) are members of the TIM family of immunomodulatory proteins [69]. They were first reported as negative regulators in Th cells that secreting IFN-g (Th1) is expressed on [70], and subsequently found it expressed on $\mathrm{DC}, \mathrm{CD} 8+\mathrm{T}$ cells and other lymphocyte subsets, and was related to its apoptosis $[71,72]$ The above shows that Tim-3 is involved in the suppression of a variety of immune cells. The overexpression of Tim-3 may lead to immunosuppression [69, 71].

Like other immunosuppressive receptors, the overexpression of Tim-3 represents a poor prognosis in solid tumors and hematological malignancies [73-75]. Researchers have found that anti-Tim-3 antibodies can improve disease progression in AML allograft models, indicating that Tim-3 is very likely to serve as an immunosuppressive marker and clinical target [76].

However, it is interesting that although Tim-3 can weaken the cytotoxicity of NK cells [77], Tim-3 also has an immune-enhancing effect, which can enhance IL-12 and IL-1 8 mediated IFN- $\gamma$ secretion by NK cells, and after Tim-3 was blocked, the production of IFN- $\gamma$ by NK cells in AML was reduced [78]. This study shows that triggering a receptor can produce two different functions. Therefore, for the Tim-3 receptor, how to use its promotion of immune function to reduce the immunosuppressive effect as much as possible is a difficult problem at present.

A study on the changes of Tim-3 expression in peripheral blood of $30 \mathrm{MM}$ patients found that the percentages of CD3+CD4+Tim-3+T and CD3+CD8+Tim-3+T cells in MM patients were higher than those in healthy subjects [79]. Tan et al. reported that in $\mathrm{MM}$, higher levels of PD1+CD244+ or 
PD1/Tim-3+CD57+CD8- T cells may be the main reason for low $\mathrm{T}$ cells activation and $\mathrm{T}$ cells immunodeficiency in $\mathrm{MM}$ patients. However, the number of Tim $-3+\mathrm{T}$ cells in different MM patients shows a high degree of individual heterogeneity, suggesting that $\mathrm{MM}$ has a complex immunosuppressive pattern [2]. Blocking negative regulatory factors including Tim-3 may further restore and release $\mathrm{T}$ cell immune function to a greater extent of MM patients.

In a mouse model, targeting Tim-3 can enhance the anti-tumor immune response by reducing Treg in head and neck squamous cell carcinoma [80]. Similarly, after the use of anti-Tim-3 antibodies in mice, it promotes anti-tumor immunity mediated by $\mathrm{T}$ cells and interferon INF- $\gamma$, and inhibits the growth of existing tumors [81]. Anti-Tim-3 antibody and anti-PD1 antibody have a synergistic effect in animal models of melanoma, which can reverse tumor-induced $\mathrm{T}$ cell failure and dysfunction in patients with advanced melanoma [82]. The above experiments have proved that after blocking Tim-3, part of the immune function can be restored and the growth of tumors can be restricted, and the combined use of anti-PD-1 antibody can enhance the effect of blocking immune suppression. Therefore, for MM tumors that also have multiple immunosuppressive molecules involved in negative regulation, combination therapy may also bring a good therapeutic response.

Currently, in the study of anti-TIM-3 antibodies, the first reported data of TSR-022 (humanized anti-TIM-3IgG4 antibody), 31 patients treated with monotherapy are in stable condition and are currently undergoing phase II clinical trials. Others, MBG453, Spartalizumab (NCT02608268), and humanized IgG4mAb, respectively block the binding of TIM-3 to PtdSer and PD- 1 to PD-L1/2, it was found in the phase I/II study of metastatic solid tumors that patients are well tolerated. Other bispecific antibodies that target both Tim- 3 and PD-1 have also entered phase I clinical trials. Whether targeted blocking tim-3 will benefit MM patients in the real world remains to be seen through the results of multiple clinical trials.

\subsection{LIRB1}

Leukocyte immunoglobulin-like receptor B1 (LILRB1) is an immune receptor based on tyrosine inhibitory motifs, which is widely expressed in human immune cells, including $\mathrm{B}$ cells, monocytes, macrophages, DC cells, and NK cells and $\mathrm{T}$ cell subsets. After binding to its ligand MHC I molecules, it activates LILRB1 and transmits inhibitory signals, thereby suppressing the immune response [8]. Studies have reported that overexpression of LILRB1 has been found in solid tumors, such as on metastatic prostate cancer [83] and breast cancer [84] the percentage of LILRB1+ in peripheral blood NK cells of patients is significantly higher than that from healthy donors or local cancer patients' NK cells.

After searching the literature of multiple myeloma and fingdingthat during the treatment of $\mathrm{MM}$ patients, the percentage of LILRB1+NK cells in the peripheral blood was significantly higher than that of healthy blood donors and patients with mild or complete remission [85]. Obviously, the overexpression of LIRBL1 in MM, is likely to be related to the severity of the patient's condition. Subsequently, experiments have proved the new anti-LILRB1 monoclonal antibody (B1-176) can block the activation of LILRB1 on NK cells by MHC-I ligands in vivo or vitro, and can stimulate NK cells killing activity against MM, leukemia and solid tumor cells [85]. Although trials have found that treatments targeting LILRB1 can show a certain effect of restoring tumor immunity, complex results have emerged. Some trials have confirmed that the absence of malignant plasma cell immune checkpoint CD85j/LILRB1 leads to immune escape in multiple myeloma, and increase the expression of LILRB1 [86]. The above studies suggest that for malignant plasma cells transformed by immune cells, such as MM LILRB+ cells, the expression of LILRB1 may inhibit the proliferation of tumor cells. This suggests that we cannot easily block the expression of LILRB1, otherwise there may be the possibility of promoting tumor cell proliferation. Therefore, for immunotherapy, patients with immune cells LILRB1+ and MM cells LILRB1- should be selected for LILRB1 receptor blocking therapy.

In addition to studying the function of LILRB1 itself, researchers have also found that human CD45RA+CCR7-CD8+ T cells are highly sensitive to BiTE molecule stimulation, but on that cells express a variety of unique inhibitory receptors, including LILRB1. The binding of LILRB1 and its ligands to tumor cells significantly inhibited the activation of $\mathrm{CD} 8+\mathrm{T}$ cells induced by BiTE molecules [87]. Therefore, blocking the expression of LILRBI can enhance the activation of BiTE molecules on CD8+ T cells. Preclinical studies have demonstrated that the use of a bi-specific $\mathrm{T}$ cell binding (BiTE) molecule combined with the TCR complex to stimulate the cytolytic activity of tumor-targeting polyclonal $\mathrm{T}$ cells has been shown to be effective in the treatment of MM [88]. The above studies have expanded our understanding of immune response, suggesting that we should not only pay attention to the direct inhibitory effect of immunosuppressive receptors on immune cells, but also pay attention to its indirect inhibitory effect. More importantly, in addition to staying in the tumor microenvironment, we should also pay attention to the role of immunosuppressive receptors expressed on tumor cells transformed from immune cells.

At present, the research results of the LILRB1 antibody have not been published. More preclinical studies are needed to provide reliable evidence in the future to further apply the anti-LILRB1 antibody to the clinic.

\section{Summary}

As the second most common hematopoietic tumor, multiple myeloma is still an incurable disease. How to prolong the survival time of patients and reduce many adverse reactions caused by treatment is a thorny issue at present. Immunotherapy has been shown to be effective in 
the treatment of solid tumors and lymphoma with relatively few adverse reactions. However, no satisfactory results have been obtained in immunotherapy for multiple myeloma. Various studies have found that several different immunosuppressive molecules play a role in the immunosuppressive mechanism of MM. However, various side effects that follow, such as cardiotoxicity [89], pulmonary toxicity [90] and so on caused by immune checkpoint inhibitor therapy, are often limits the application of immune checkpoint inhibitors in clinical settings. At present, the understanding of immunosuppressive receptors in $\mathrm{MM}$ is insufficient. This review summarized part of the studies on PD-1, TCLA-4, Tim-3, TIGIT and other popular immunosuppressive receptors in $\mathrm{MM}$, in order to attract more attention, and in-depth research on the immunotherapy also to promote the immunotherapy of MM from basic research to clinical transformation as soon as possible.

\section{Statement}

The manuscript has been read and approved by all the authors.

\section{Conflict of Interest}

All the authors do not have any possible conflicts of interest.

\section{References}

[1] Mortaz E, Tabarsi P, Mansouri D, et al. Cancers Related to Immunodeficiencies: Update and Perspectives. Front Immunol. 2016; 7: 365 .

[2] Tan J, Chen S, Huang J, et al. Increased exhausted CD8+T cells with programmed death-1, T-cell immunoglobulin and mucin-domain-containing-3 phenotype in patients with multiple myeloma. Asia-Pacific Journal of Clinical Oncology. 2018; 14 (5): e266-e74.

[3] Cooke RE, Quinn KM, Quach H, et al. Conventional Treatment for Multiple Myeloma Drives Premature Aging Phenotypes and Metabolic Dysfunction in T Cells. Front Immunol. 2020; 11: 2153.

[4] Elhelbawy NG, Nassar AAH, Eltorgoman AEA, et al. Immunological microenvironment gene expression in patients with diffuse large B cell non Hodgkin lymphoma. Biochem Biophys Rep. 2020; 21: 100731.

[5] Meng F, Li L, Lu F, et al. Overexpression of TIGIT in NK and $\mathrm{T}$ Cells Contributes to Tumor Immune Escape in Myelodysplastic Syndromes. Front Oncol. 2020; 10: 1595.

[6] Panda A, Rosenfeld JA, Singer EA, et al. Genomic and immunologic correlates of LAG-3 expression in cancer. Oncoimmunology. 2020; 9 (1): 1756116.

[7] Jones RB, Ndhlovu LC, Barbour JD, et al. Tim-3 expression defines a novel population of dysfunctional $\mathrm{T}$ cells with highly elevated frequencies in progressive HIV-1 infection. J Exp Med. 2008; 205 (12): 2763-79.
[8] Kang X, Kim J, Deng M, et al. Inhibitory leukocyte immunoglobulin-like receptors: Immune checkpoint proteins and tumor sustaining factors. Cell Cycle. 2016; 15 (1): 25-40.

[9] Takahashi T TT, Yamazaki S, et al.. Immunologic self-tolerance maintained by CD25 $(+) \mathrm{CD} 4(+)$ regulatory $\mathrm{T}$ cells constitutively expressing cytotoxic $\mathrm{T}$ lymphocyte-associated antigen 4.. journal of Experiment Medicine. 2000.

[10] Liu X, Ranganathan R, Jiang S, et al. A Chimeric Switch-Receptor Targeting PD1 Augments the Efficacy of Second-Generation CAR T Cells in Advanced Solid Tumors. Cancer Res. 2016; 76 (6): 1578-90.

[11] Yan Z, Yao ZH, Yao SN, et al. Combining PD-1 Inhibitor Nivolumab with Radiotherapy Successfully Treated a Patient with Refractory Primary Mediastinal Large B-Cell Lymphoma: A Case Report and Literature Review. Cancer Manag Res. 2020; 12: 6311-6.

[12] Pan B, Shang L, Liu C, et al. PD-1 antibody and ruxolitinib enhances graft-versus-lymphoma effect without increasing acute graft-versus-host disease in mice. Am J Transplant. 2020 .

[13] Hui E, Cheung J, Zhu J, et al. T cell costimulatory receptor CD28 is a primary target for PD-1-mediated inhibition. Science. 2017; 355 (6332): 1428-33.

[14] Francisco LM, Sage PT, Sharpe AH. The PD-1 pathway in tolerance and autoimmunity. Immunol Rev. 2010; 236: 219-42.

[15] Tan J, Chen S, Lu Y, et al. Higher PD-1 expression concurrent with exhausted CD8+ $\mathrm{T}$ cells in patients with de novo acute myeloid leukemia. Chin J Cancer Res. 2017; 29 (5): 463-70.

[16] Passiglia F, Bironzo P, Righi L, et al. A Prospective Phase II Single-arm Study of Niraparib Plus Dostarlimab in Patients With Advanced Non-small-cell Lung Cancer and/or Malignant Pleural Mesothelioma, Positive for PD-L1 Expression and Germline or Somatic Mutations in the DNA Repair Genes: Rationale and Study Design. Clin Lung Cancer. 2020.

[17] Willenbacher W, Willenbacher E, Zelle-Rieser C, et al. Bone marrow microenvironmental CD4 + and CD8 + lymphocyte infiltration patterns define overall- and progression free survival in standard risk multiple myeloma--an analysis from the Austrian Myeloma Registry. Leuk Lymphoma. 2016; 57 (6): $1478-81$.

[18] Li Y, Chen S, Yang L, et al. Change in expression pattern of TCR-CD3 complex in patients with multiple myeloma. Hematology. 2011; 16 (3): 143-50.

[19] Gordon SR, Maute RL, Dulken BW, et al. PD-1 expression by tumour-associated macrophages inhibits phagocytosis and tumour immunity. Nature. 2017; 545 (7655): 495-9.

[20] Ray A, Das DS, Song Y, et al. Targeting PD1-PDL1 immune checkpoint in plasmacytoid dendritic cell interactions with $\mathrm{T}$ cells, natural killer cells and multiple myeloma cells. Leukemia. 2015; 29 (6): 1441-4.

[21] Benson DM, Jr., Bakan CE, Mishra A, et al. The PD-1/PD-L1 axis modulates the natural killer cell versus multiple myeloma effect: a therapeutic target for CT-011, a novel monoclonal anti-PD-1 antibody. Blood. 2010; 116 (13): 2286-94. 
[22] Hallett WH, Jing W, Drobyski WR, et al. Immunosuppressive effects of multiple myeloma are overcome by PD-L1 blockade. Biol Blood Marrow Transplant. 2011; 17 (8): 1133-45.

[23] Lesokhin AM, Ansell SM, Armand P, et al. Nivolumab in Patients With Relapsed or Refractory Hematologic Malignancy: Preliminary Results of a Phase Ib Study. J Clin Oncol. 2016; 34 (23): 2698-704.

[24] Badros A, Hyjek E, Ma N, et al. Pembrolizumab, pomalidomide, and low-dose dexamethasone for relapsed/refractory multiple myeloma. Blood. 2017; 130 (10): 1189-97.

[25] Rizvi NA, Hellmann MD, Snyder A, et al. Mutational landscape determines sensitivity to PD-1 blockade in non-small cell lung cancer. Science. 2015; 348 (6230): 124-8.

[26] Tumeh PC, Harview CL, Yearley JH, et al. PD-1 blockade induces responses by inhibiting adaptive immune resistance. Nature. 2014; 515 (7528): 568-71.

[27] Herbst RS, Soria JC, Kowanetz M, et al. Predictive correlates of response to the anti-PD-L1 antibody MPDL3280A in cancer patients. Nature. 2014; 515 (7528): 563-7.

[28] Braga WMT, da Silva BR, de Carvalho AC, et al. FOXP3 and CTLA4 overexpression in multiple myeloma bone marrow as a sign of accumulation of CD4+ $\mathrm{T}$ regulatory cells. Cancer Immunology, Immunotherapy. 2014; 63 (11): 1189-97.

[29] Zhu Y, Wu S. [Immune Characteristics of Small Cell Lung Cancer]. Zhongguo Fei Ai Za Zhi. 2020; 23 (10): 889-96.

[30] Gao X, Yang J, Chen Y. Identification of a four immune-related genes signature based on an immunogenomic landscape analysis of clear cell renal cell carcinoma. J Cell Physiol. 2020; 235 (12): 9834-50.

[31] Aref S, El Agdar M, El Sebaie A, et al. Prognostic Value of CD200 Expression and Soluble CTLA-4 Concentrations in Intermediate and High-Risk Myelodysplastic Syndrome Patients. Asian Pac J Cancer Prev. 2020; 21 (8): 2225-30.

[32] Tcvetkov N, Gusak A, Morozova E, et al. Immune checkpoints bone marrow expression as the predictor of clinical outcome in myelodysplastic syndrome. Leuk Res Rep. 2020; 14: 100215.

[33] Hodi FS ODS, McDermott DF, et al. Improved survival with ipilimumab in patients with metastatic melanoma. he New England journal of medicine. 2010.

[34] Chau V, Bilusic M. Pembrolizumab in Combination with Axitinib as First-Line Treatment for Patients with Renal Cell Carcinoma (RCC): Evidence to Date. Cancer Manag Res. 2020; 12: 7321-30.

[35] Pinheiro FD, Teixeira AF, de Brito BB, et al. Immunotherapy new perspective in lung cancer. World J Clin Oncol. 2020; 11 (5): 250-9.

[36] Allison MFKaJp. CD28 and CTLA-4 Have Opposing Effects on the Response of $\mathrm{T}$ ceils to Stimulation. Journal of Experiment Medicine. 1995.

[37] Brian T. JAB. Control of peripheral T-cell tolerance and autoimmunity via the CTLA-4 and PD-1 pathways. Bluestone - Immunological reviews. 2008.

[38] Zhang H, Dutta P, Liu J, et al. Tumour cell-intrinsic CTLA4 regulates PD-L1 expression in non-small cell lung cancer. J Cell Mol Med. 2019; 23 (1): 535-42.
[39] Hellmann MD, Rizvi NA, Goldman JW, et al. Nivolumab plus ipilimumab as first-line treatment for advanced non-small-cell lung cancer (CheckMate 012): results of an open-label, phase 1, multicohort study. The Lancet Oncology. 2017; 18 (1): $31-41$.

[40] Postow MA, Chesney J, Pavlick AC, et al. Nivolumab and ipilimumab versus ipilimumab in untreated melanoma. $\mathrm{N}$ Engl J Med. 2015; 372 (21): 2006-17.

[41] Tang J, Chen Q, Zhang F, et al. [Peripheral blood exosomes from patients with multiple myeloma mediate bortezomib resistance in cultured multiple myeloma cells]. Nan Fang Yi Ke Da Xue Xue Bao. 2019; 39 (4): 485-9.

[42] Chalmers AW, Patel S, Boucher K, et al. Phase I Trial of Targeted EGFR or ALK Therapy with Ipilimumab in Metastatic NSCLC with Long-Term Follow-Up. Target Oncol. 2019; 14 (4): 417-21.

[43] Dougall WC, Kurtulus S, Smyth MJ, et al. TIGIT and CD96: new checkpoint receptor targets for cancer immunotherapy. Immunological Reviews. 2017; 276 (1): 112-20.

[44] Guillerey C, Ferrari de Andrade L, Vuckovic S, et al. Immunosurveillance and therapy of multiple myeloma are CD226 dependent. J Clin Invest. 2015; 125 (5): 2077-89.

[45] Hoogi S, Eisenberg V, Mayer S, et al. A TIGIT-based chimeric co-stimulatory switch receptor improves T-cell anti-tumor function. J Immunother Cancer. 2019; 7 (1): 243.

[46] Kitsou M, Ayiomamitis GD, Zaravinos A. High expression of immune checkpoints is associated with the TIL load, mutation rate and patient survival in colorectal cancer. Int J Oncol. 2020; 57 (1): 237-48.

[47] Xu D, Zhao E, Zhu C, et al. TIGIT and PD-1 may serve as potential prognostic biomarkers for gastric cancer. Immunobiology. 2020; 225 (3).

[48] Simone A. Minnie RDK, Kate H. Gartlan, Ping Zhang, Andrew N. Wilkinson. Myeloma escape after stem cell transplantation is aconsequence of T-cell exhaustion and is prevented by TIGIT blockade. blood. 2018.

[49] Guillerey C, Harjunpaa H, Carrie N, et al. TIGIT immune checkpoint blockade restores CD8 (+) T-cell immunity against multiple myeloma. Blood. 2018; 132 (16): 1689-94.

[50] Johnston RJ, Comps-Agrar L, Hackney J, et al. The immunoreceptor TIGIT regulates antitumor and antiviral CD8 (+) T cell effector function. Cancer Cell. 2014; 26 (6): 923-37.

[51] Zhang Q, Bi J, Zheng X, et al. Blockade of the checkpoint receptor TIGIT prevents NK cell exhaustion and elicits potent anti-tumor immunity. Nat Immunol. 2018; 19 (7): 723-32.

[52] He W, Zhang H, Han F, et al. CD155T/TIGIT Signaling Regulates CD8 (+) T-cell Metabolism and Promotes Tumor Progression in Human Gastric Cancer. Cancer Res. 2017; 77 (22): 6375-88.

[53] Wang J, Sanmamed MF, Datar I, et al. Fibrinogen-like Protein 1 Is a Major Immune Inhibitory Ligand of LAG-3. Cell. 2019; $176(1-2)$ : 334-47 e12.

[54] Turnis ME, Andrews LP, Vignali DA. Inhibitory receptors as targets for cancer immunotherapy. Eur J Immunol. 2015; 45 (7): 1892-905. 
[55] Zarour HM. Reversing T-cell Dysfunction and Exhaustion in Cancer. Clin Cancer Res. 2016; 22 (8): 1856-64.

[56] Chen PL, Roh W, Reuben A, et al. Analysis of Immune Signatures in Longitudinal Tumor Samples Yields Insight into Biomarkers of Response and Mechanisms of Resistance to Immune Checkpoint Blockade. Cancer Discov. 2016; 6 (8): 827-37.

[57] Andrews LP, Marciscano AE, Drake CG, et al. LAG3 (CD223) as a cancer immunotherapy target. Immunol Rev. 2017; 276 (1): 80-96.

[58] Kim YJ, Won CH, Lee MW, et al. Correlation Between Tumor-Associated Macrophage and Immune Checkpoint Molecule Expression and Its Prognostic Significance in Cutaneous Melanoma. J Clin Med. 2020; 9 (8).

[59] Saleh R, Taha RZ, Toor SM, et al. Expression of immune checkpoints and $\mathrm{T}$ cell exhaustion markers in early and advanced stages of colorectal cancer. Cancer Immunol Immunother. 2020; 69 (10): 1989-99.

[60] Radwan SM, Elleboudy NS, Nabih NA, et al. The immune checkpoints Cytotoxic $\mathrm{T}$ lymphocyte antigen-4 and Lymphocyte activation gene-3 expression is up-regulated in acute myeloid leukemia. HLA. 2020; 96 (1): 3-12.

[61] Xie N, Sun YY, Jiang HJ, et al. [Lymphocyte activation gene 3 expression on $\mathrm{T}$ lymphocyte cell subsets in patients with myelodysplastic syndrome]. Zhonghua Yi Xue Za Zhi. 2020; 100 (24): 1886-9.

[62] Mussetti A, Pellegrinelli A, Cieri N, et al. PD-L1, LAG3, and HLA-DR are increasingly expressed during smoldering myeloma progression. Ann Hematol. 2019; 98 (7): 1713-20.

[63] Lucas F, Pennell M, Huang Y, et al. T Cell Transcriptional Profiling and Immunophenotyping Uncover LAG3 as a Potential Significant Target of Immune Modulation in Multiple Myeloma. Biol Blood Marrow Transplant. 2020; 26 (1): 7-15.

[64] Grosso JF, Kelleher CC, Harris TJ, et al. LAG-3 regulates $\mathrm{CD} 8+\mathrm{T}$ cell accumulation and effector function in murine self- and tumor-tolerance systems. J Clin Invest. 2007; 117 (11): 3383-92.

[65] Deng WW, Mao L, Yu GT, et al. LAG-3 confers poor prognosis and its blockade reshapes antitumor response in head and neck squamous cell carcinoma. Oncoimmunology. 2016; 5 (11): e1239005.

[66] Yu X, Huang X, Chen X, et al. Characterization of a novel anti-human lymphocyte activation gene 3 (LAG-3) antibody for cancer immunotherapy. MAbs. 2019; 11 (6): 1139-48.

[67] Jing W, Gershan JA, Weber J, et al. Combined immune checkpoint protein blockade and low dose whole body irradiation as immunotherapy for myeloma. J Immunother Cancer. 2015; 3 (1): 2.

[68] Legat A, Maby-El Hajjami H, Baumgaertner $P$, et al. Vaccination with LAG-3Ig (IMP321) and Peptides Induces Specific CD4 and CD8 T-Cell Responses in Metastatic Melanoma Patients--Report of a Phase I/IIa Clinical Trial. Clin Cancer Res. 2016; 22 (6): 1330-40.

[69] Freeman GJ, Casasnovas JM, Umetsu DT, et al. TIM genes: a family of cell surface phosphatidylserine receptors that regulate innate and adaptive immunity. Immunol Rev. 2010; 235 (1): 172-89.
[70] Zhu C, Anderson AC, Schubart A, et al. The Tim-3 ligand galectin-9 negatively regulates $\mathrm{T}$ helper type 1 immunity. Nat Immunol. 2005; 6 (12): 1245-52.

[71] Anderson AC AD, Bregoli L, Hastings WD, Kassam N, Lei C, Chandwaskar R, Karman J, Su EW, Hirashima M, Bruce JN, Kane LP, Kuchroo VK, Hafler DA. Promotion of Tissue Inflammation by the Immune Receptor Tim-3 Expressed on Innate Immune Cells. Science 2007 Nov 16. 2007.

[72] Nakayama M, Akiba H, Takeda K, et al. Tim-3 mediates phagocytosis of apoptotic cells and cross-presentation. Blood. 2009; 113 (16): 3821-30.

[73] Holderried TAW, de Vos L, Bawden EG, et al. Molecular and immune correlates of TIM-3 (HAVCR2) and galectin 9 (LGALS9) mRNA expression and DNA methylation in melanoma. Clin Epigenetics. 2019; 11 (1): 161.

[74] Jia K, He Y, Dziadziuszko R, et al. T cell immunoglobulin and mucin-domain containing-3 in non-small cell lung cancer. Transl Lung Cancer Res. 2019; 8 (6): 895-906.

[75] Tan J, Yu Z, Huang J, et al. Increased PD-1+Tim-3+ exhausted $\mathrm{T}$ cells in bone marrow may influence the clinical outcome of patients with AML. Biomarker Research. 2020; 8 (1).

[76] Kikushige Y, Shima T, Takayanagi S, et al. TIM-3 is a promising target to selectively kill acute myeloid leukemia stem cells. Cell Stem Cell. 2010; 7 (6): 708-17.

[77] Ndhlovu LC, Lopez-Verges S, Barbour JD, et al. Tim-3 marks human natural killer cell maturation and suppresses cell-mediated cytotoxicity. Blood. 2012; 119 (16): 3734-43.

[78] Gleason MK, Lenvik TR, McCullar V, et al. Tim-3 is an inducible human natural killer cell receptor that enhances interferon gamma production in response to galectin-9. Blood. 2012; 119 (13): 3064-72.

[79] Hui Liu J-QZ, Zeng-Feng Ni, Yu-Hong Liu, Jiao Jiao Correlation of the Expression Alteration of Tim-3 with the T Cell and B Cell Dysfunction in Peripheral Blood of Multiple Myeloma Patients.

[80] Zhongguo Shi Yan Xue Ye Xue Za Zhi 2020 Jun; 28 (3): 881-885 Chinese. 2020.

[81] Liu JF, Wu L, Yang LL, et al. Blockade of TIM3 relieves immunosuppression through reducing regulatory $\mathrm{T}$ cells in head and neck cancer. J Exp Clin Cancer Res. 2018; 37 (1): 44.

[82] Ngiow SF, von Scheidt B, Akiba H, et al. Anti-TIM3 antibody promotes $\mathrm{T}$ cell IFN-gamma-mediated antitumor immunity and suppresses established tumors. Cancer Res. 2011; 71 (10): 3540-51.

[83] Fourcade J, Sun Z, Benallaoua M, et al. Upregulation of Tim-3 and PD-1 expression is associated with tumor antigen-specific $\mathrm{CD} 8+\mathrm{T}$ cell dysfunction in melanoma patients. J Exp Med. 2010; 207 (10): 2175-86.

[84] Pasero C, Gravis G, Guerin M, et al. Inherent and Tumor-Driven Immune Tolerance in the Prostate Microenvironment Impairs Natural Killer Cell Antitumor Activity. Cancer Res. 2016; 76 (8): 2153-65.

[85] Roberti MP, Julia EP, Rocca YS, et al. Overexpression of CD85j in TNBC patients inhibits Cetuximab-mediated NK-cell ADCC but can be restored with CD85j functional blockade. Eur J Immunol. 2015; 45 (5): 1560-9. 
[86] Chen H, Chen Y, Deng M, et al. Antagonistic anti-LILRB1 monoclonal antibody regulates antitumor functions of natural killer cells. J Immunother Cancer. 2020; 8 (2).

[87] Lozano E, Díaz T, Mena M-P, et al. Loss of the Immune Checkpoint CD85j/LILRB1 on Malignant Plasma Cells Contributes to Immune Escape in Multiple Myeloma. The Journal of Immunology. 2018; 200 (8): 2581-91.

[88] Kim A, Han CJ, Driver I, et al. LILRB1 Blockade Enhances Bispecific T Cell Engager Antibody-Induced Tumor Cell Killing by Effector CD8 (+) T Cells. J Immunol. 2019; 203 (4): 1076-87.
[89] Goldstein RL, Goyos A, Li CM, et al. AMG 701 induces cytotoxicity of multiple myeloma cells and depletes plasma cells in cynomolgus monkeys. Blood Adv. 2020; 4 (17): 4180-94.

[90] Guo X, Wang H, Zhou J, et al. [Clinical Diagnosis and Treatment Recommendations for Cardiac Adverse Reactions Related to Immune Checkpoint Inhibitor]. Zhongguo Fei Ai Za Zhi. 2019; 22 (10): 627-32.

[91] Wang H, Guo X, Zhou J, et al. [Clinical Diagnosis and Treatment Recommendations for the Pneumonitis Associated with Immune Checkpoint Inhibitor]. Zhongguo Fei Ai Za Zhi. 2019; 22 (10): 621-6. 